\title{
Alternativas de control orgánico in vitro para Dactylonectria torresensis en la mora de castilla (Rubus glaucus) en Ecuador
}

\section{In Vitro Organic Control Alternatives for Dactylonectria torresensis in Blackberry (Rubus glaucus) in Ecuador}

\author{
Racines-Oliva, M. ${ }^{1}$, Tamayo-Gutiérrez, E. ${ }^{2}$, Jarrín, M. ${ }^{3}$, Báez, F. ${ }^{4}$, Tello, C. ${ }^{5}$
}

\begin{abstract}
Resumen
Esta investigación evaluó, en laboratorio, la eficiencia de los fungicidas de origen orgánico frente a los fungicidas químicos de uso convencional para controlar el crecimiento del Dactylonectria torresensis, considerada como una de las fitopatologías de importancia económica que afecta a la mora de castilla (Rubus glaucus) en Ecuador. El objetivo del estudio se basó en la necesidad imperante de buscar alternativas naturales y amigables con el ambiente, que permitan obtener alimentos limpios para la población. Para tal fin, se evaluó la eficiencia de los fungicidas convencionales de síntesis química: Azoxystrobin, Benomil, Carbendazim, Difeconazol, Fosetyl Aluminio, Tachigaren, Propiconazol, Penconazol, Metalaxil y Thiabendazole, cada uno en dosis de 100, 10 y 1 ppm, frente a la eficiencia de fungicidas de síntesis orgánica: aceite de neem, extracto de mirtáceas, extracto de ajo, sulfato cúplico pentahidratado y extracto de tomillo, aplicados en dosis de 300, 200, y 100 ppm. Se comprobó que el extracto de mirtáceas controló en 100 \% del crecimiento micelial del Dactylonectria torresensis, y mostró resultados similares a los obtenidos con Carbendazim y Azoxystrobin. Se concluye, por lo tanto, que si existiera una alternativa para el control del hongo, esta podría ser utilizada comercialmente, y de esta manera disminuir el uso y los efectos nocivos de los agroquímicos convencionales.
\end{abstract}

\section{Palabras clave}

Fungistático, esporulación, orgánico, Mirtaceae, micelio.

\begin{abstract}
The following research evaluated, at laboratory level, the efficiency of fungicides of organic origin against chemical fungicides of conventional use in the control of the growth of Dactylonectria torresensis, considered a pathogen of economic importance that affects the blackberry (Rubus glaucus) in Ecuador. The objective of the study was to find natural, environmentally friendly alternatives that allow obtaining clean food for the population. For this purpose, the efficiency of conventional chemical synthesis fungicides was evaluated: Azoxystrobin, Benomil, Carbendazim, Difeconazole, Fosetyl Aluminum, Tachigaren, Propiconazole, Penconazole, Metalaxil and Thiabendazole each in doses of 100, 10 and $1 \mathrm{ppm}$, compared to the efficiency of organic synthesis fungicides: neem oil, mirtaceous extract, garlic extract, pentahydrate cupric sulfate, thyme extract, applied in doses of 100, 200, and $300 \mathrm{ppm}$. It was found that the mirtaceous extract controlled $100 \%$ of the mycelial growth of Dactylonectria torresensis, with results similar to those obtained with Carbendazim and Azoxystrobin, it is concluded that if there is an alternative for the control of the fungus, which could be used commercially by decreasing in this way the use and harmful effects of conventional agrochemicals.
\end{abstract}

\section{Keywords}

Fungistatic; sporulation; organic; Mirtaceae; mycelium.

\section{Introducción}

La mora de castilla (Rubus glaucus) originaria de la región Andina constituye un frutal de alta importancia económica en Ecuador, debido a la demanda creciente y la rentabilidad del cultivo 
(INIAP, 2016). Actualmente, este fruto tiene potencial de exportación tanto fresco como transformado en productos alimenticios, industrialmente (Saltos, 2001). Su consumo se diversifica desde fruta fresca hasta la elaboración de productos con valor agregado como mermeladas, pulpas, colorantes, entre otros. En Ecuador, el cultivo de la mora de castilla se encuentra ubicado a lo largo del Callejón Interandino, a una altitud de 1200 y 3000 msnm (Bejarano, 1992); y, de acuerdo con datos publicados por el MAGAP (2013), el 70 \% de la producción nacional se encuentra en la provincia de Tungurahua.

Debido a los cambios bruscos de temperatura, el cultivo de la mora tiene alta susceptibilidad a las afecciones de origen fúngico, de estas la más relevante es el pie negro. El agente causal del pie negro de la mora de Castilla estaba identificado como Cylindrocarpon destructans, pero recientemente se determinó que la enfermedad es causada por un grupo de hongos de los géneros Ilyonectria y Dactylonectria, incluido el Dactylonectria torresensis, hongo mitospórico cuya característica es la presencia de fiálidas largas y conidios hialinos (Sánchez et al., 2019).

El pie negro de la mora es una enfermedad que se identifica por la reducción del vigor, necrosis en el tejido vascular, clorosis, pudrición del cuello y raíces y ausencia de brotes en la planta; puede además causar la muerte de la planta (Leiva, 2011). La incidencia y severidad de esta afección han generado cambios en cuanto al manejo fitosanitario en el cultivo de la mora, que lo hace más agresivo y constante (Alarcón, J., Garrido, L., y Leiva, L. , 2011).

Esta práctica ha causado el incremento del uso de agroquímicos para manejar los cultivos convencionales y evitar la merma producida por este patógeno (Martínez, 2014). Sin embargo, esto ha incidido de forma perjudicial en la cadena de valor de la mora, lo que causa que el fruto llegue al mercado con altos contenidos de residuos de agroquímicos, y que se reduzcan las oportunidades de exportación por no alcanzar los parámetros mínimos exigidos a nivel internacional (Corporación Colombiana de Investigación Agropecuaria, 2008).

En este marco, se están generando esfuerzos en el ámbito de la investigación en laboratorio y en campo como aporte para proponer alternativas viables, funcionales y económicas a los productores con el fin de obtener cultivos inocuos con base en una 'agricultura limpia'.

Devia (2011) define la agricultura limpia como "[...] una forma de producción agropecuaria que aplica procedimientos especiales que buscan proteger la naturaleza y sus especies". Actualmente, el auge del consumo más saludable se ha incrementado a nivel mundial, y existe una concienciación sobre reducir químicos en toda la gama alimenticia desde la producción de materia prima. Por esta razón, el objetivo de la investigación fue evaluar, en condiciones controladas de laboratorio, la eficiencia de fungicidas de origen orgánico frente a fungicidas de síntesis química de uso convencional, para controlar el crecimiento micelial del hongo Dactylonectria torresensis, aislado de la mora de castilla producida en la provincia de Tungurahua.

Como hipótesis se estableció que al menos un fungicida orgánico presenta eficiencia in vitro semejante a los fungicidas químicos para controlar el hongo.

\section{Metodología}

La investigación tomó lugar en el laboratorio de Fitopatología del Departamento de Protección Vegetal del Instituto Nacional de Investigaciones Agropecuarias (INIAP), en Quito, Ecuador, en condiciones de altitud de $3058 \mathrm{msnm}$, longitud $78^{\circ} 33^{\prime} \mathrm{O}$ y latitud $00^{\circ} 22^{\prime} \mathrm{S}$.

El estudio se desarrolló en dos etapas. La primera sirvió para la evaluación in vitro del efecto fungistático de cada uno de los productos evaluados, y el efecto sobre la capacidad 
esporulación del hongo Dactylonectria torresensis en condiciones controladas de humedad del $80 \%$, temperatura de $18{ }^{\circ} \mathrm{C}$ y sin luz. En la segunda etapa se consideró calcular la concentración efectiva (EC50) del agroquímico de interés.

En la investigación se aplicó un diseño al azar en arreglos factoriales de 10×3+1 para los tratamientos con productos de síntesis química, y $5 \times 3+1$ para los de síntesis orgánica. Se obtuvo un total de 47 tratamientos correspondientes a las interacciones de los factores más un testigo absoluto, con diez repeticiones para cada uno. Estos tratamientos se describen en las tablas 1 y 2 :

Tabla 1. Descripción de los tratamientos de estudio para evaluar los fungicidas de síntesis química

\begin{tabular}{|c|c|c|}
\hline Codificación & Fungicida & Dosis ppm \\
\hline c1d1 & Benomil & 1 \\
\hline $\mathrm{c} 1 \mathrm{~d} 2$ & Benomil & 10 \\
\hline c1d3 & Benomil & 100 \\
\hline c2d1 & Carbendazim & 1 \\
\hline $\mathrm{c} 2 \mathrm{~d} 2$ & Carbendazim & 10 \\
\hline $\mathrm{c} 2 \mathrm{~d} 3$ & Carbendazim & 100 \\
\hline c3d1 & Fosetyl Aluminio & 1 \\
\hline $\mathrm{c} 3 \mathrm{~d} 2$ & Fosetyl Aluminio & 10 \\
\hline c3d3 & Fosetyl Aluminio & 100 \\
\hline c4d1 & Metalaxil & 1 \\
\hline $\mathrm{c} 4 \mathrm{~d} 2$ & Metalaxil & 10 \\
\hline c4d3 & Metalaxil & 100 \\
\hline c5d1 & Thiabendazole & 1 \\
\hline $\mathrm{c} 5 \mathrm{~d} 2$ & Thiabendazole & 10 \\
\hline $\mathrm{c} 5 \mathrm{~d} 3$ & Thiabendazole & 100 \\
\hline c6d1 & Difeconazol & 1 \\
\hline $\mathrm{c} 6 \mathrm{~d} 2$ & Difeconazol & 10 \\
\hline c6d3 & Difeconazol & 100 \\
\hline c7d1 & Himexazol & 1 \\
\hline c7d2 & Himexazol & 10 \\
\hline c7d3 & Himexazol & 100 \\
\hline c8d1 & Propiconazol & 1 \\
\hline $\mathrm{c} 8 \mathrm{~d} 2$ & Propiconazol & 10 \\
\hline c8d3 & Propiconazol & 100 \\
\hline c9d1 & Penconazol & 1 \\
\hline $\mathrm{c} 9 \mathrm{~d} 2$ & Penconazol & 10 \\
\hline $\mathrm{c9d3}$ & Penconazol & 100 \\
\hline c10d1 & Azoxystrobin & 1 \\
\hline c10d2 & Azoxystrobin & 10 \\
\hline c10d3 & Azoxystrobin & 100 \\
\hline Testigo absoluto & No contiene & $\mathrm{n} / \mathrm{a}$ \\
\hline
\end{tabular}

Fuente: INIAP, 2016. 
Tabla 2. Descripción de los tratamientos de estudio para evaluar los fungicidas de síntesis orgánica

\begin{tabular}{|l|l|l|}
\hline \multicolumn{1}{|c|}{ Codificación } & \multicolumn{1}{|c|}{ Fungicida } & \multicolumn{1}{c|}{ Dosis ppm } \\
\hline a1d1 & Extracto de mirtáceas & 1000 \\
\hline a1d2 & Extracto de mirtáceas & 2000 \\
\hline a1d3 & Extracto de mirtáceas & 3000 \\
\hline a2d1 & Aceite de neem & 1000 \\
\hline a2d2 & Aceite de neem & 2000 \\
\hline a2d3 & Aceite de neem & 3000 \\
\hline a3d1 & Sulfato cúprico pentahidratado & 1000 \\
\hline a3d2 & Sulfato cúprico pentahidratado & 2000 \\
\hline a3d3 & Sulfato cúprico pentahidratado & 3000 \\
\hline a4d1 & Extracto de ajo & 1000 \\
\hline a4d2 & Extracto de ajo & 2000 \\
\hline a4d3 & Extracto de ajo & 3000 \\
\hline a5d1 & Extracto de tomillo & 1000 \\
\hline a5d2 & Extracto de tomillo & 2000 \\
\hline a5d3 & Extracto de tomillo & 3000 \\
\hline
\end{tabular}

Fuente: INIAP, 2016

\section{1. Aislamiento y purificación de Dactylonectria torresensis}

Antes de la evaluación de los fungicidas, se aisló al patógeno tomando muestras del tejido vegetal infectado con una superficie aproximada de $0.5 \mathrm{~cm}^{2}$, las cuales fueron desinfectadas con hipoclorito al $1 \%$, por 2 minutos, y luego lavadas tres veces con agua destilada esterilizada (ADE). Se colocaron las muestras en cajas de Petri con Potato Dextrose Agar (PDA) para incubarlas a una temperatura de $18{ }^{\circ} \mathrm{C}$, durante un período de siete días, y transcurrido ese tiempo fueron purificadas (Cedeño, Carrero, Quintero, Pino y Espinoza, 2004).

\subsection{Manejo de fungicidas y preparación de agares}

El manejo del fungicida se llevó a cabo por el método de diluciones en plato de agar (Rondón, Sanabría y Rondón, 2006), en el que se preparó una solución madre concentrada a 1000 ppm con ADE. A partir de esta se dispusieron diluciones específicas de $10 \mathrm{ml}$ mezcladas con $90 \mathrm{ml}$ del medio de cultivo PDA, en condiciones de temperatura de $45^{\circ} \mathrm{C}$. Este medio preparado se dispuso en las cajas de Petri de $9 \mathrm{~cm}$ de diámetro (Camele et al., 2012).

Para evaluar la eficiencia del fungicida, se tomaron discos de micelio de $5 \mathrm{~mm}$ de diámetro de un cultivo aislado del patógeno y se los depositó en el centro de la caja de Petri. La caja contenía el medio de cultivo más el fungicida de cada uno de los tratamientos, que fueron incubados a $21^{\circ} \mathrm{C}$ por períodos de siete y catorce días, para luego medir los diámetros de crecimientos de la colonia en cm (Cedeño et al., 2004).

Para la evaluación estadística, se aplicó la prueba de Tukey al 5 \% para fungicida, dosis e interacción. 


\subsection{Efecto fungistático de los productos evaluados}

Se calculó el porcentaje de inhibición mediante el método detallado por Vincent, (1947) que relaciona los datos del crecimiento radial con el control y los datos del crecimiento radial en el tratamiento por medio de la siguiente fórmula:

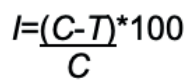

I= Porcentaje de inhibición,

$C=$ Crecimiento radial del control $(\mathrm{cm}), \mathrm{y}$

$T=$ Crecimiento radial del tratamiento $(\mathrm{cm})$

\subsection{Capacidad de esporulación}

A partir del tratamiento fungistático, a los catorce días de cultivo fueron recolectadas muestras de $5 \mathrm{~mm}^{2}$ del centro de las cajas de Petri, se las colocó en tubos Eppendorf con $1 \mathrm{ml}$ de ADE y se las expuso a agitación. Finalmente, se contabilizó el número de conidias con un hematocitómetro (Viera, 2002).

\subsection{Cálculo del EC50}

El EC50 constituye la concentración de los productos que inhibe el crecimiento del patógeno en un 50 \%. Esta variable se precisó con la metodología de Probit, que involucra el valor del crecimiento del micelio en porcentaje, y tiene como referente el crecimiento de un testigo absoluto frente a las concentraciones de los tratamientos (Viera, 2002). Para esta evaluación fueron tomados los datos de los siete fungicidas con mayor efectividad en las evaluaciones previas.

\section{Resultados}

El resultado base del cual proviene la comparativa entre los distintos tratamientos estuvo dado por el crecimiento micelial del testigo absoluto, el cual ha sido considerado en porcentaje al 100 $\%$. De esta estimación fueron obtenidos los resultados subsiguientes detallados a continuación.

\subsection{Resultado de la evaluación del efecto fungistático: productos de síntesis química}

Esta evaluación reflejó que los resultados obtenidos en el séptimo día de incubación no mostraron diferencias significativas entre los tratamientos en contraste con los resultados de la toma de muestra al decimocuarto día. En este tiempo la diferencia entre los crecimientos miceliales fue muy evidente, por lo tanto, se corroboró lo citado por (Gaviria-Hernández, Patiño y Saldarriaga, 2013) que el factor tiempo es determinante en cuanto al desarrollo del hongo en el medio de cultivo. En tal virtud, los resultados que se presentan a continuación son los evaluados únicamente durante el decimocuarto día de cultivo.

En la tabla 3 se registran los datos obtenidos del efecto fungistático de los tratamientos a las diferentes concentraciones. Hay que tomar en cuenta que mayor eficiencia mostrarán los resultados cuya media sea más cercana a uno. 
Tabla 3. Efecto fungistático (\%) de los productos de síntesis química en el control de Dactylonectria torresensis en mora de castilla

\begin{tabular}{|l|l|l|l|}
\hline \multirow{2}{*}{\multicolumn{1}{c|}{ Fungicida }} & \multicolumn{2}{c|}{ Dosis } \\
\cline { 2 - 4 } & \multicolumn{1}{c|}{$\mathbf{1}$ ppm } & \multicolumn{1}{c|}{$10 \mathrm{ppm}$} & \multicolumn{1}{c|}{$100 \mathrm{ppm}$} \\
\hline Carbendazim & $1 \pm 0.00 \mathrm{D}$ & $1 \pm 0.00 \mathrm{E}$ & $1 \pm 0.00 \mathrm{C}$ \\
\hline Azoxystrobin & $1 \pm 0.00 \mathrm{D}$ & $1 \pm 0.00 \mathrm{E}$ & $1 \pm 0.00 \mathrm{C}$ \\
\hline Propiconazol & $0.71 \pm 0.03 \mathrm{C}$ & $0.82 \pm 0.04 \mathrm{D}$ & $1 \pm 0.00 \mathrm{C}$ \\
\hline Difeconazol & $0.58 \pm 0.04 \mathrm{~B}$ & $0.74 \pm 0.05 \mathrm{CD}$ & $1 \pm 0.00 \mathrm{C}$ \\
\hline Penconazol & $0.53 \pm 0.05 \mathrm{~B}$ & $0.67 \pm 0.05 \mathrm{C}$ & $1 \pm 0.00 \mathrm{C}$ \\
\hline Benomil & $0.21 \pm 0.06 \mathrm{~A}$ & $0.67 \pm 0.05 \mathrm{C}$ & $1 \pm 0.00 \mathrm{C}$ \\
\hline Himexazol & $0.22 \pm 0.04 \mathrm{~A}$ & $0.34 \pm 0.05 \mathrm{~B}$ & $0.67 \pm 0.05 \mathrm{~B}$ \\
\hline Metalaxil & $0.17 \pm 0.07 \mathrm{~A}$ & $0.14 \pm 0.10 \mathrm{~A}$ & $1 \pm 0.00 \mathrm{C}$ \\
\hline Thiabendazole & $0.15 \pm 0.10 \mathrm{~A}$ & $0.63 \pm 0.13 \mathrm{C}$ & $1 \pm 0.00 \mathrm{C}$ \\
\hline Fosetyl Aluminio & $0.1 \pm 0.09 \mathrm{~A}$ & $0.16 \pm 0.05 \mathrm{~A}$ & $0.18 \pm 0.09 \mathrm{~A}$ \\
\hline
\end{tabular}

Como se puede apreciar en la Tabla 3, la eficiencia fungistática está liderada por los productos Carbendazim y Azoxystrobin, los cuales, independientemente de las dosis a las cuales fueron aplicados, muestran un valor de media de 1 \pm 0.00 ; mientras que los fungicidas Himexazol y Fosetil Aluminio son los agroquímicos que tienen medias más bajas en todos los tratamientos evaluados.

\subsection{Resultados de la evaluación del efecto fungistático: productos de síntesis orgánica}

Asimismo, los datos se tomaron en el decimocuarto día de incubación. Los resultados obtenidos en las mediciones se los puede ver en la Tabla 4:

Tabla 4. Efecto fungistático (\%) de los productos de síntesis orgánica en el control de Dactylonectria torresensis en mora de castilla

\begin{tabular}{|l|l|l|l|}
\hline \multirow{2}{*}{\multicolumn{1}{c|}{ Fungicida }} & \multicolumn{2}{c|}{ Dosis } & \multicolumn{1}{c|}{$300 \mathrm{ppm}$} \\
\cline { 2 - 4 } & \multicolumn{1}{c|}{$\mathbf{1 0 0} \mathbf{~ p p m}$} & \multicolumn{1}{c|}{$\mathbf{2 0 0} \mathrm{ppm}$} & $1 \pm 0.00 \mathrm{D}$ \\
\hline Extracto de mirtáceas & $0.8 \pm 0.00 \mathrm{C}$ & $0.9 \pm 0.00 \mathrm{C}$ & $1 \pm 0.00 \mathrm{D}$ \\
\hline Extracto de ajo & $0.14 \pm 0.05 \mathrm{~A}$ & $0.14 \pm 0.05 \mathrm{~A}$ & $0.85 \pm 0.05 \mathrm{C}$ \\
\hline Sulfato cúprico pentahidratado & $0.82 \pm 0.04 \mathrm{C}$ & $0.8 \pm 0.00 \mathrm{C}$ & $0.34 \pm 0.05 \mathrm{~B}$ \\
\hline Aceite de neem & $0.33 \pm 0.05 \mathrm{~B}$ & $0.31 \pm 0.03 \mathrm{~B}$ & $0.17 \pm 0.07 \mathrm{~A}$ \\
\hline Extracto de tomillo & $0.15 \pm 0.05 \mathrm{~A}$ & $0.17 \pm 0.07 \mathrm{~A}$ & \\
\hline
\end{tabular}

Se resalta como resultado de interés que el extracto de mirtáceas refleja una media cercana a uno en todas las dosis evaluadas y alcanza un valor máximo de $1 \pm 0.00$ en la dosis de 300 ppm. Respecto al extracto de ajo, este muestra un valor de media de $1 \pm 0.00$ solo en la dosis de 300 ppm, y en dosis menores no refleja un efecto fungistático eficiente. Para finalizar, el sulfato cúprico pentahidratado presenta medias con valores de entre 0.8 y 0.85 que pueden considerarse aceptables en la eficiencia del control fungistático del hongo. 


\subsection{Resultados de la evaluación de la concentración de los productos frente a la esporulación}

Como primer resultado, se presenta que el conteo de unidades formadoras de colonias (UFC) representadas por el número de conidias para el testigo absoluto estuvo dado por $1389400 \pm 95972.91$.

Los resultados de la medición de esporulación de los diferentes tratamientos en estudio, sin embargo, mostraron gran variabilidad entre ellos. Las medias de la contabilización de UFC se registraron en la Tabla 5 para los tratamientos de los fungicidas de síntesis química y, en la Tabla 6, los tratamientos de sintesis orgánica.

Tabla 5. Unidades formadoras de colonias (UFC) de Dactylonectria torresensis por efecto de los productos de síntesis química

\begin{tabular}{|l|l|l|l|}
\hline \multirow{2}{*}{\multicolumn{1}{c|}{ Fungicida }} & \multicolumn{2}{c|}{ Dosis } \\
\cline { 2 - 4 } & \multicolumn{1}{c|}{$1 \mathrm{cpm}$} & \multicolumn{1}{c|}{$10 \mathrm{ppm}$} & \multicolumn{1}{c|}{$100 \mathrm{ppm}$} \\
\hline Benomil & $65000.00 \pm 13674.79 \mathrm{~A}$ & $0.00 \pm 0.00 \mathrm{~A}$ & $0.00 \pm 0.00 \mathrm{~A}$ \\
\hline Carbendazim & $0.00 \pm 0.00 \mathrm{~A}$ & $0.00 \pm 0.00 \mathrm{~A}$ & $0.00 \pm 0.00 \mathrm{~A}$ \\
\hline Fosetyl Aluminio & $0.00 \pm 0.00 \mathrm{~A}$ & $0.00 \pm 0.00 \mathrm{~A}$ & $0.00 \pm 0.00 \mathrm{~A}$ \\
\hline Metalaxil & $0.00 \pm 0.00 \mathrm{~A}$ & $84400.00 \pm 7569.68 \mathrm{CDE}$ & $40800.00 \pm 2489.98 \mathrm{ABC}$ \\
\hline Thiabendazole & $38600.00 \pm 2190.89 \mathrm{~A}$ & $0.00 \pm 0.00 \mathrm{~A}$ & $0.00 \pm 0.00 \mathrm{~A}$ \\
\hline Difeconazol & $107600.00 \pm 42618.07 \mathrm{~A}$ & $0.00 \pm 0.00 \mathrm{~A}$ & $107600.00 \pm 42618.07 \mathrm{DE}$ \\
\hline Himexazol & $134400.00 \pm 3646.92 \mathrm{E}$ & $131200.00 \pm 6978.54 \mathrm{E}$ & $11800.00 \pm 47058.47 \mathrm{DE}$ \\
\hline Propiconazol & $21000.00 \pm 8124.04 \mathrm{~A}$ & $0.00 \pm 0.00 \mathrm{~A}$ & $0.00 \pm 0.00 \mathrm{~A}$ \\
\hline Penconazol & $103000.00 \pm 41551.17 \mathrm{~A}$ & $0.00 \pm 0.00 \mathrm{~A}$ & $103000.00 \pm 41551.17 \mathrm{DE}$ \\
\hline Azoxystrobin & $0.00 \pm 0.00 \mathrm{~A}$ & $0.00 \pm 0.00 \mathrm{~A}$ & $0.00 \pm 0.00 \mathrm{~A}$ \\
\hline
\end{tabular}

Tabla 6. Unidades formadoras de colonias (UFC) de Dactylonectria torresensis por efecto de los productos de síntesis orgánica

\begin{tabular}{|l|l|l|l|}
\hline \multirow{2}{*}{\multicolumn{1}{c|}{ Fungicida }} & \multicolumn{2}{c|}{ Dosis } & \multicolumn{1}{c|}{$300 \mathrm{ppm}$} \\
\cline { 2 - 4 } & \multicolumn{1}{|c|}{$100 \mathrm{ppm}$} & \multicolumn{1}{c|}{$\mathbf{p p m}$} & \multicolumn{1}{c|}{$300 \mathrm{Ep}-11 \pm 0.00 \mathrm{~A}$} \\
\hline Extracto de mirtáceas & $5.80 \mathrm{E}-11 \pm 0.00 \mathrm{~A}$ & $1.20 \mathrm{E}-10 \pm 0.00 \mathrm{~A}$ & $57600 \pm 10945.32 \mathrm{~A}$ \\
\hline Aceite de neem cúprico & $60800 \pm 10756.39 \mathrm{~A}$ & $58200 \pm 10084.4 \mathrm{~A}$ & $300000 \pm 4123.11 \mathrm{~B}$ \\
\hline $\begin{array}{l}\text { Sulfato } \\
\text { pentahidratado }\end{array}$ & $298600 \pm 6228.96 \mathrm{~B}$ & $300200 \pm 2558.44 \mathrm{C}$ & $0.00 \pm 0.00 \mathrm{~A}$ \\
\hline Extracto de ajo & $3.94 \mathrm{E}+04 \pm 2073.64 \mathrm{~A}$ & $3.92 \mathrm{E}+4 \pm 836.66 \mathrm{~A}$ & $69800 \pm 2387.47 \mathrm{~A}$ \\
\hline Extracto de tomillo & $502400 \pm 10454.66 \mathrm{C}$ & $207600 \pm 8876.94 \mathrm{~B}$ & \\
\hline
\end{tabular}

Se puede observar que los tratamientos de sintesis químico pertenecientes al grupo estadístico A controlan de manera eficiente el crecimiento de unidades formadoras de colonias; mientras que en los fungicidas de síntesis orgánica el extracto de ajo induce a un conteo de esporulación de cero a una dosis de concentración de 300 ppm; por lo tanto, es un resultado óptimo para controlar las esporas del patógeno. 


\subsection{Resultado del cálculo de la concentración efectiva EC50}

Este cálculo se lo aplicó en siete fungicidas de mayor eficacia comprobada en los resultados previamente expuestos: Benomil, Difeconazol, Penconazol, Propiconazol, Thiabendazole, extracto de mirtáceas y extracto de ajo. Como valor promedio del cálculo de EC50 de los fungicidas químicos, se obtuvo un valor de $55 \mathrm{ppm}$, sin que muestre diferencia significativa entre los resultados de sus pares. Los fungicidas orgánicos alcanzaron un valor promedio de EC50 de 2500 ppm.

\section{Discusión}

El crecimiento del micelio del patógeno en estudio estuvo influenciado por el factor tiempo, resultado similar a la premisa explicada por Ferro (2008), lo que permitió que se descarten los primeros resultados obtenidos al séptimo día de incubación del hongo y que se consideren los que fueron tomados el decimocuarto día de incubación.

Rego, Farropas, Nascimento, Cabral y Oliveira (2006) y Halleen, Fourie y Crous (2007) presentan evaluaciones previas para el uso de fungicidas de síntesis química en el control de Cylindrocarpon spp., lo que determina que los fungicidas Carbendazim (clorhidrato de carbendacima), Procloraz (N-propil-N-[2-(2,4,6-triclorofenoxi)etil]imidazol-1-carboxamida), Tebuconazol ((RS)-1-p-chlorophenyl-4,4-dimethyl-3-(1H-1,2,4-triazol-1-ylmethyl)pentan-3-ol) muestran eficiencia marcada en su control; lo que se pudo confirmar en esta investigación, en la cual se obtuvieron valores similares.

Benalcázar (2011) demuestra también en su estudio que los fungicidas Carberndazim más Tebuconazol inhiben el crecimiento micelial de Sphaeroteca pannosa en un $100 \%$ de eficiencia. Este dato se utilizó de referente para comprobar su amplitud de eficiencia en el control fitosanitario con los agroquímicos utilizados en campo actualmente.

Los resultados del control fungistático de los tratamientos con productos de síntesis química demostraron que la eficiencia del control estuvo relacionada directamente proporcional con la concentración del tratamiento, lo que concuerda con los estudios de Viera (2002) y Benalcázar (2011), en los cuales se presenta que la eficiencia de los fungicidas sistémicos incrementaron su control en dosis altas; y donde los Benzimidazoles lideraron los resultados. Respecto del caso específico de control de Dactylonectria torresensis, el que presentó mayor eficacia fue el Carbendazim.

Para analizar el control de la esporulación, los resultados obtenidos en el estudio concuerdan de manera similar con los de Viera (2002), en cuanto a la existencia de fungicidas de síntesis químicas que resultan ineficientes para controlar la esporulación, premisa que se evidencia en las tablas de resultados. Esto es fundamental, pues la reproducción de patógenos puede ser muy virulenta si no existe control efectivo, y, por lo tanto, la aplicación de los productos químicos en los campos para controlar el patógeno se incrementa sin obtener un resultado favorable.

El potencial antifúngico de los productos de síntesis orgánica que fueron seleccionados para este estudio basa su eficacia en los estudios de Farag (1989) y Nychas (1995). Los autores atribuyen esta característica a la presencia de compuestos monoterpénicos y fenólicos, que pueden tener la capacidad de atacar a la membrana del citoplasma del hongo y afectar de manera sustancial a la capacidad selectiva de este, además de poder inactivar las enzimas responsables del desarrollo fúngico. 
En contraste con los estudios de Hernández, Bautista y Velázquez (2007), Müller-Riebau, Berger y Yegen (1995) y Plotto, Roberts y Roberts (2003), quienes presentan en sus estudios la eficiencia en el control de Alternaria arborescens, Botrytis cinérea, Phytopthora capsici y Rhizopus stolonifer con el extracto de tomillo (Timol), para este estudio, la utilización del mismo producto para controlar el Dactylonectria torresensis no fue efectiva. Esto se evidenció con los valores obtenidos tanto en el cálculo de inhibición fungistática como en el conteo de unidades formadoras de colonias, en el que se obtuvo valores inferiores a los de otros tratamientos como los desarrollados a base de extracto de mirtáceas y de extracto de ajo.

Al relacionar los resultados obtenidos entre la eficiencia del producto de síntesis química con el producto de síntesis orgánica, existen diferencias significativas. Sin embargo, se destaca que existen dos productos orgánicos relevantes que actúan de manera considerable sobre el efecto inhibitorio del fitopatógeno en estudio: el extracto de mirtáceas y sulfato cúprico pentahidratado. Adicionalmente, en los resultados obtenidos en el estudio del efecto sobre la esporulación del hongo, el extracto de ajo también mostró resultados favorables.

\section{Conclusiones y recomendaciones}

Se concluye que sí existen alternativas de productos de síntesis orgánica que puedan controlar eficientemente el crecimiento y esporulación de Dactylonectria torresensis en laboratorio; estos son el extracto de mirtáceas, el sulfato cúprico pentahidratado y el extracto de ajo.

Los resultados obtenidos en esta investigación presentan un panorama esperanzador en cuanto al desarrollo de métodos amigables con el ambiente, que puedan ser utilizados en campo con el fin de disminuir el uso de agentes nocivos para la salud y aportar a la producción limpia de alimentos.

Se recomienda desarrollar investigaciones en campo sobre la efectividad del uso de los productos mencionados, así como también se sugiere ampliar las opciones de dosis de aplicación para que se pueda considerar con datos más certeros el cálculo del EC50.

\section{Agradecimientos}

Agradecemos a AgResearh por el aporte económico que permitió el desarrollo de este trabajo de investigación.

Agradecemos especialmente a los organizadores del ॥ Simposio Internacional de Producción Integrada de Frutas 2019, quienes han permitido que los avances de la investigación científica en el área de frutales puedan llegar a los productores, lo que provee de alternativas que contribuyen de manera significativa en la generación de recursos económicos y en el mejoramiento de la calidad de vida de los pobladores de nuestro país.

\section{Bibliografía}

Alarcón, J., Garrido, L., y Leiva, L. (2011). Manejo fitosanitario del cultivo de mora (Rubus glaucus benth). ICA. Recuperado de http://www.ica.gov.co/getattachment/b7e061eb-ebd3-4f80-9518c771712405eb/-nbsp;Manejo-fitosanitario-del-cultivo-de-la-mora.aspx

Bejarano, W. (1992). Manual de mora: (Rubus claucus Beth). Quito, Ecuador: Proexant.

Benalcázar, V. (2011). Evaluación in vitro en laboratorio de seis fungicidas con diferentes mecanismos de acción, como alternativa para el control de oidio (Sphaeroteca pannosa) en el cultivo aislado 
de rosas en el cantón Ibarra, provincia de Imbabura. Pontificia Universidad Católica del Ecuador. Recuperado de: dspace.pucesi.edu.ec/bitstream/11010/166/1/T72594.pdf

Camele, I.; Altieri, L.; De Martino, L.; De Feo, V.; Mancini, E., y Rana, G. (2012). In Vitro Control of Post-Harvest Fruit Rot Fungi by Some Plant Essential Oil Components. International Journal of Molecular Science, 13(2), 2290-300.

Cedeño, L.; Carrero, C.; Quintero, K.; Pino, H., y Espinoza, W. (2004). Cylindrocarpon destructans and Neonectrina discophora var. Rubi Associated with Black Foot Rot on Blackberry (Rubus glaucus Benth) in Merida, Venezuela, Interciencia, 29(8), 455-460.

Corporación Colombiana de Investigación Agropecuaria. (2008). Compilación: Tecnología para la producción de frutales de clima frío moderado. Estación experimental La Suiza. Corpoica-libra. Rionegro-Santander-Colombia.

Devia, C. (2011). Agricultura limpia: Sistemas y prácticas de producción bajo el concepto de agricultura limpia. Recuperado de http://www.asohofrucol.com.co/archivos/biblioteca/2Agricultura\%20 limpia.pdf

Farag, K. (1989). Enhancing Ethephon Effectiveness by Modifyung Cuticular Transport of Stimulating Ethylene Production in Cranberry Fi-Uit (Tesis de doctorado). University de Wisconsisn-Madison, Estados Unidos.

Ferro, S. (2008). Caracterización de Cylindrocarpon spp., agente causal del pie negro en la vid (p. 181), (Tesis doctoral). Universidad Politécnica de Valencia.

Gaviria-Hernández, V.; Patiño, L., y Saldarriaga, E. (2013). Evaluación in vitro de fungicidas comerciales para el control de Colletotrichum spp., en mora de castilla. Ciencia y Tecnología Agropecuaria,14(1), 67-75.

Halleen, F.; Fourie, P., y Crous, P. (2007). Control of Black Foot Disease in Grapenive Nurseries. Plant Pathology, 56(4) 637-645.

Hernández A.; Bautista, S., y Velázquez, M. (2007). Prospectiva de extractos vegetales para controlar enfermedades poscosecha hortofrutícolas. Revista Fitotecnia Mexicana, 30(2), 119-123.

INIAP. (2016). El cultivo de la mora en el Ecuador. Recuperado de http://repositorio.iniap.gob.ec/bitstream/41000/4878/1/iniapsc355.pdf

Leiva, L. (2011). Manejo fitosanitario de la mora. Produmedios. Colombia

MAGAP. (2013). La mora de castilla. Características de la mora de castilla. MAGAP, 5-18.

Martínez, D. (2014). Identificación de hongos fitopatógenos relacionados con la marchitez de mora de castilla (Rubus glaucus Benth) en la provincia de Tungurahua mediante microscopía óptica y PCR (Tesis de pregrado). Universidad de las Fuerzas Armadas, Ecuador.

Müller-Riebau, M.; Berger, B., y Yegen, O. (1995). Chemical Composition and Fungitoxic Properties of Phytopathogenic Fungi of Essential Oils Selected Aromatic Plants Growing Wild in Turkey. Journal of Agricultural and Food Chemistry 43(8), 2262-2266.

Nychas, G. (1995). Natural Antimicrobials from Plants. En Gloud, G. W. (ed.). New Methods of Food Preservation, Cap. 4 (pp. 59-89). Londres, Reino Unido: Aspen Publishers.

Plotto, A.; Roberts, D., y Roberts, R. (2003). Evaluation of Plant Essential Oils as Natural Postharvest Disease Control of Tomato (Lycopersicon esculentum). Acta Horticulturae, 628, 737-745.

Rego, C.; Farropas, L.; Nascimento, T.; Cabral, A., y Oliveira, H. (2006). Black Foot of Grapevine: Sensivity of Cylindrocarpon destructans to Fungicides. Phytopathología Mediterránea, 45(4), S93-S100.

Rondón, O.; Sanabría, N., y Rondón, A. (2006). Respuesta in vitro a la acción de fungicidas para el control de antracnosis, Colletotrichum gloeosporioides Penz, en frutos de mango. Agronomía Tropical, 56(2), 219-235. Recuperado de http://www.scielo.org.ve/scieldo.php?script=sci_ arttext\&pid=S0002-192X2006000200005\&lng=es\&tlng=es

Saltos, A. (2001). Investigación y desarrollo de tecnologías aplicadas a la conservación de frutas-mora de castilla (Rubus glaucus Benth), UTA-BID-FUNDACYT, Ambato, Ecuador. 
Sánchez, J.; Iturralde, P.; Koch, A.; Tello, C.; Martínez, D.; Proaño, N.; Martínez, A., Viera, W.; Ayala, L., y Flores, F. (2019). Dactylonectria and Ilyonectria Species Causing Black Foot Disease of Andean Blackberry (Rubus Glaucus Benth) in Ecuador. Diversity, 11(11), 218.

Viera, W. (2002). Evaluación de fungicidas in vitro y pruebas de resistencia de cinco variedades de tomates de árbol (Solanum betaceum Cav.) para antracnosis (Colletotrichum gloeosporoides) (Tesis de grado). Universidad Central del Ecuador, Ecuador .

Vincent, J. (1947). Distortion of fungal hyphae in the Presence of Certain Inhibitors. Nature, 159(850). 